\title{
An Improved Algorithm for Indirect Time domain Analysis of Thin Wire Structures
}

\author{
Siniša Antonijević, Vicko Dorić, and Dragan Poljak
}

\begin{abstract}
The algorithm for an indirect time domain (TD) analysis using arbitrary frequency domain method is presented. In previous work, an optimized version of the indirect timedomain method based on adaptive sampling iterative algorithm used in combination with inverse Fast Fourier Transform (FFT) was presented. In this paper, the algorithm is further improved by circumventing the use of inverse FFT altogether, and using the simple formula based on the analytical inverse Fourier transform. The proposed approach is simpler and more convenient for use with the underlying iterative optimization method, as only a part of the frequency domain (FD) signal has to be transformed to the time domain during each iteration procedure, the frequency domain discretisation does not have to be uniform, and the time domain discretisation does not have to be performed. These advantages can also lead to the reduced computational time cost of the frequency to time domain transformation when compared with the standard inverse FFT.
\end{abstract}

Index Terms-time domain analysis, optimization, adaptive sampling, inverse Fast Fourier Transform, analytical Fourier transform.

\section{INTRODUCTION}

Transient analysis of thin wire structures can be generally performed via direct and indirect time domain methods [1]. Direct time domain methods are formulated and solved directly in the time domain, while indirect methods use frequency domain as a basis for the formulation, as well as for obtaining the set of frequency domain solutions. Thus obtained solutions need to be transformed into the time domain via some appropriate transformation technique, typically inverse Fast Fourier Transform (FFT). Indirect time domain methods are therefore in principle easier to formulate and implement than direct time domain methods, which makes them more often used than direct time domain methods [1][2]. By using the well tested frequency domain solver, such as Numerical Electromagnetic Code (NEC) [3], as a basis for the indirect time domain analysis in combination with FFT, a reliable benchmark time domain results can be achieved [4]-

Manuscript received December, 2011.

The material in this paper was presented in part at the 19th International Conference on Software, Telecommunications and Computer Networks (SoftCOM 2011), Split-Hvar-Dubrovnik, Croatia, Sept. 2011.

Siniša Antonijević, Vicko Dorić, and Dragan Poljak are with the University of Split, Split, Croatia (e-mail: santon@pmfst.hr)
[6]. On the other hand, for geometries which impulse response spans across larger frequency spectrum, or that are highly resonant, indirect methods are significantly less efficient than direct time domain methods, which require only one calculation, regardless of the observed geometry [1]-[2]. In this regard, additional advantage of the direct time domain methods is that transient response of the observed structure is usually of interest only in the early time interval, where the transient non-harmonic behavior is typically most pronounced. Indirect methods yield a transient response for all times, not only for the early time periods, which is also one of the reasons of their relative computational inefficiency when compared to the direct time domain methods.

However, this problem can be significantly alleviated if uniform sampling of current where frequency domain solutions to be calculated is avoided. The basic idea is to perform the calculation only for the frequencies which significantly contribute to the corresponding time domain behavior of the system transient response, while other frequencies (with low amplitude harmonics or slowly changing transfer function of the system) can be interpolated. Typical examples of such an approach are algorithms focusing on the resonant frequencies or poles of the frequency domain solution, resulting in higher density of samples around the resonant frequencies [7]-[9]. Similar approach was adopted in the algorithm presented in [10], where it was shown that more than an order of magnitude improvements of the overall computation time can be achieved using simple iterative adaptive sampling algorithm for finding significant resonant frequencies, and using simple linear interpolation for frequencies that are not sampled. In this paper, the algorithm presented in [10] is further improved by using analytical inverse Fourier transform. The usage of high enough number of points in inverse FFT can be circumvented altogether and the unknown current in the frequency domain can be approximated as a series of known functions spanned by the points determined during the adaptive sampling step of the algorithm. In this way, a significantly smaller number of samples need to be used in order to calculate the time domain inverse of the frequency domain current, by subjecting the approximate function to the analytical inverse Fourier transform. The resulting function is a sum of simple closed form expressions that can readily be numerically evaluated. This procedure also has an additional advantage that the complete inversion, over the entire frequency span, does not 
need to be repeated in the each iteration. In other words, all summands need not need not be evaluated, but only those pertaining to the additional segments determined by the adaptive sampling algorithm. The principal advantage of this approach, when compared to the usage of FFT is its formulation simplicity and implementation when applied to the iterative adaptive sampling algorithm.

The layout of the paper is as follows: Chapter 2 outlines the basic algorithm presented in [10], and in Chapter 3 the implementation of the analytical inverse Fourier Transform on the previously described algorithm is presented. Numerical examples illustrating the method are given in Chapter 4, followed by Conclusion.

\section{II.INDIRECT TIME-DOMAIN ANALYSIS USING ADAPTIVE SAMPLING ALGORITHM}

The procedure of calculating time-domain results via indirect method based on NEC can be divided in several basic steps. First, viewing the geometry as a linear system, transfer function of the analyzed geometry $H(f)$ is obtained by executing NEC sufficient number of times so that $H(f)$ curve is accurate enough. If excitation with magnitude 1 and phase 0 is used for all frequencies, the obtained frequency domain current results represent the transfer function of the system. Then, time-dependant voltage source $V(t)$ is sampled and FFT is performed, resulting in a frequency spectrum of the excitation $V(f)$. Next, excitation frequency spectrum $V(f)$ and structure transfer function $H(f)$ are multiplied in the frequency domain thus obtaining frequency-domain current distribution on the observation point at the analyzed structure $I(f)=H(f) V(f)$. Finally, $I(t)$ is calculated from $I(f)$ via the inverse FFT.

The principal disadvantage of this procedure, with regards to computational time, is that the frequency domain calculation has to be repeated sufficient number of times in order to obtain satisfactory accuracy of the results - that is, time domain current. Namely, $H(f)$ have to be sampled with sufficient density of samples. If the analyzed structure is highly resonant, there will be very pronounced and narrow high magnitude peaks in $H(f)$ function, that have to be sampled very densely. If the simplest, uniform sampling is used, there will be very high number of samples for such structures, and the overall calculation time will get unacceptably long. This follows from the basic parameters of the sampling process in inverse Discrete Fourier Transform (DFT) [11]. Given some maximum frequency of the transfer function $f_{\max }$, representing the bandwidth of the $I(f)$, and the number of frequency domain samples $N$, the frequency domain sampling interval $\Delta f$ is given by:

$$
\Delta f=\frac{f_{\max }}{N} .
$$

Time domain sampling interval $\Delta t$ is related to $f_{\max }$ according to the Nyquist sampling theorem which states that the time domain rate of sampling $f_{s}=1 / \Delta t$ should be at least twice the bandwidth of the signal. Therefore, the maximum time domain sampling interval is

$$
\Delta t=\frac{1}{2 f_{\max }} .
$$

If Nyquist sampling condition, represented by (2) is not satisfied, i.e. if $\Delta t$ is choosen larger then value specified by (2), aliasing errors will be introduced in the time domain results.

Since time domain and frequency domain number of samples must be the same $(N)$, maximum observation time period $T$ is than simply $T=\Delta t \cdot N$, or using relations (1) and (2):

$$
T=\frac{N}{2 f_{\max }}=\frac{1}{2 \Delta f} .
$$

Therefore, with relations (1)-(3) all the parameters of the inverse FFT are completely determined, once $f_{\max }$ and $N$ are chosen. Obviously, the larger the values of these parameters, the better the results, but the question is how to determine their minimum acceptable value. These parameters can not be determined in advance, since the $I(t)$ and therefore $I(f)$ are unknown, so initially $f_{\max }$ and $N$ have to be chosen empirically. However, there are some strategies that can help in rough $a$ priori estimation of $f_{\max }$. For example, at $f_{\max }$ magnitudes of the $I(f)$ drop to sufficiently small values, so that they can be considered zero. Although $H(f)$ is unknown, since it depends on the specific geometry that is being analyzed, the frequency counterpart $V(f)$ of the excitation (forcing) function $V(t)$ is known, and its bandwidth can be used instead. If $f_{\max }$ is chosen in such a way that $V\left(f_{\max }\right)$ becomes sufficiently small, so that it can be considered zero, $I\left(f_{\max }\right)$ can also be considered zero (assuming $H\left(f_{\max }\right)$ is not very large), since $I(f)=V(f) H(f)$ [7], [12], [13]. Another approach is applicable in case a more simple geometries, such as single wires, are analyzed. In this case, resonant frequencies are approximately [14]-[16]

$$
f_{0 i}=i \frac{c}{2 L} ; \quad i=1,2, \ldots
$$

where $c$ is the speed of light, and $L$ is the length of the wire. It is therefore important to choose $f_{\max }$ larger than at least first resonant frequency [15].

Number of samples $N$ should also be chosen sufficiently large, so that the transfer function of the geometry is sampled accurately enough in the frequency domain, especially around the resonant frequencies which appear as peaks in the transfer function $H(f)$. If those frequencies are not sampled, an important frequency domain behavior information is skipped, and the resulting time domain results will be highly inaccurate. Also, choosing an insufficiently high value of $N$ will, according to (1) and (3), result in larger $\Delta f$ and therefore 
smaller $T$. Too small observation period $T$ will in turn cause truncation of the time domain results, and end-effect error, causing the time domain waveform truncated end to appear "wrapped around" on the starting point at $t=0$ [8].

One, rather obvious, solution to the problem of choosing both $f_{\text {max }}$ and $N$ high enough, without proportional increase in computational cost, is selectively increasing the sampling density only around resonant frequencies. However, if no $a$ priori knowledge about the waveform of the frequency domain response is assumed, the problem is related to efficient assessment of the resonant frequencies. This problem can then be tackled by using some adaptive sampling algorithm. Generally, the basic idea of adaptive sampling is to construct iterative algorithm for selecting the frequencies that are most likely to be near the resonant frequencies, based on the results from the previous iterations. One such algorithm was presented in [8], and in this chapter, a basic outline of this algorithm is presented.

The algorithm can be described in several fundamental steps:

1. Initial guess: select initial small set of frequencies

2. Frequency domain calculation of the current for each frequency on a given frequency set

3. Obtain time-domain current using inverse FFT

4. Decide if time domain current is accurate enough - if yes, go to step 7

5. Based on the frequency domain results obtained so far, find a new frequency set to be calculated

6. Go to step 2

7. End

Initially, an arbitrary set of frequencies is chosen in order to obtain a very rough estimate of the transfer function. If no previous knowledge about the frequency domain structure behavior is assumed, then a straightforward choice is to simply use a small set of frequencies uniformly distributed from zero to some maximum frequency. In the next step, frequency domain calculation is repeated for all of the chosen frequencies. This can be undertaken by any frequency domain method suitable for the analysis of the observed geometry - in this paper the well known Numeric Electromagnetic Code is used as a frequency domain solver. Although the third step can be avoided, the intermediate time-domain results can be convenient in construction of the stopping criteria, as described in [8]. Inspection of the intermediate time-domain results is also useful in case the iteration is to be stopped by hand, prior to fulfillment of the stopping criteria in the fourth step.

The crux of the algorithm is in the fifth step. The question is how to decide what frequencies to choose in the next iteration. The algorithm should choose those frequencies where the impact of the error between approximated and the actual transfer function on the shape of the resultant time domain current is expected to be the greatest. Obviously, the best candidates for these are the resonant frequencies. The behavior of the transfer function $H(f)$ around the resonant frequencies will manifest itself as a rapid change in the curve representing the absolute value of $H(f)$. So, the basic idea is to choose the frequencies where the rate of change of the $|H(f)|$ is the greatest. However, in case the magnitude of $|H(f)|$ is very small, even in case the curve is very steep, the frequencies with much greater magnitudes of $|H(f)|$ will have a more significant influence on the final time domain results. It follows that the criteria for choosing the frequencies to be calculated in the next iteration should take into account both the rate of change and the magnitude of the transfer function around each of the frequencies calculated in the previous iterations.

This criteria can be described by a very simple relation assigning an error estimate $e_{i}$ to each of the frequencies calculated so far. The error $e_{i}$ for $\underline{i}$-th point is constructed by taking into account neighboring points.

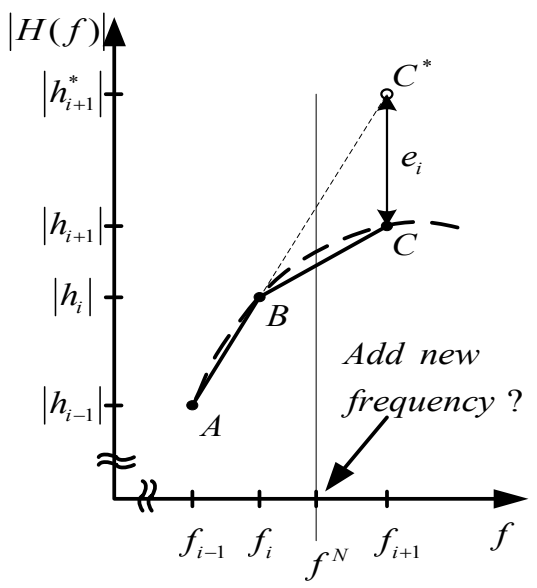

Fig. 1. Interpolation error estimation for frequency $f_{i}$ : new frequency $f^{N}$ is added only if $e_{i}$ is large enough

As illustrated in Fig. 1, the value of estimated error $e_{i}$ represents the difference between magnitude at the frequency $f_{i+1}$ (point $\mathrm{C}$ ), and extrapolated value (point $\mathrm{C}^{*}$ ), based on points $\mathrm{A}$ and $\mathrm{B}$ :

$$
e_{i}=|| h_{i}\left|+\frac{f_{i+1}-f_{i}}{f_{i}-f_{i-1}}\left(\left|h_{i}\right|-\left|h_{i-1}\right|\right)-\right| h_{i+1}|| .
$$

If point $\mathrm{B}$ is the first point that was calculated $(i=1)$, the previous point (point A) is assigned zero value, since in this case $f_{i-1}=0$, and therefore $H(0)=0$. The new frequency points are added only for frequencies larger then the first frequency $f_{1}$, so $f_{1}$ should be chosen small enough during the first step, i.e. the initial frequency selection.

Estimating the errors for each pair $\left(f_{i}, h_{i}\right)$, only frequencies with estimated error $e_{i}$ greater then some minimum value $e_{\text {min }}$ are chosen as a reference for adding a new frequency, and new frequencies to be calculated in next iteration are chosen halfway between $f_{i}$ and $f_{i+1}$, as illustrated in Fig. 1 . The value of $e_{\min }$ is chosen so that in the next iteration, only the points 
with associated above average error estimations are used for calculation of the new frequencies. This can be determined using the standard deviation of all estimated errors:

$$
e_{\min }=\bar{e}+K \sigma ; \quad K \in \mathbb{R}
$$

where

$$
\begin{gathered}
\sigma=\sqrt{\frac{1}{N} \sum_{i=1}^{N}\left(e_{i}-\bar{e}\right)^{2}}, \\
\bar{e}=\frac{1}{N} \sum_{i=1}^{N} e_{i},
\end{gathered}
$$

$N$ is the total number of frequencies calculated up to the current iteration and $K$ is empirical coefficient, typically chosen between 1 and 1,5.

\section{ANALYTICAL INVERSE FOURIER TRANSFORM OF THE APPROXIMATE FUNCTION}

Instead of using inverse FFT and resampling the transfer function to obtain time domain results, a simple method, that requires only samples acquired during adaptive sampling estimation in previous iteration, can be used. The basic idea is to use simple linear interpolation to approximate the segment of the unknown $I(f)$ function as an analytical function fully described by the two neighboring samples:

$$
\tilde{I}_{k}(f)=I_{k} \frac{f_{k+1}-f}{f_{k+1}-f_{k}}+I_{k+1} \frac{f-f_{k}}{f_{k+1}-f_{k}} .
$$

Fig. 2. depicts a general case where the unknown current can be approximated by linear function along the segment $k$, bracketed by the frequencies $f_{k}$ and $f_{k+1}$ :

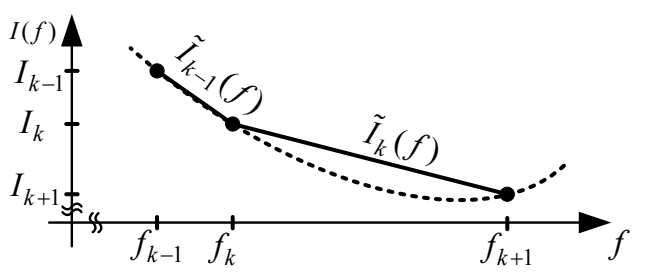

Fig. 2. Linear interpolation of the FD current

Expression (9) can be rewritten as

$$
\tilde{I}_{k}(f)=A_{k} f+B_{k},
$$

where

$$
A_{k}=\frac{I_{k+1}-I_{k}}{f_{k+1}-f_{k}} ; \quad B_{k}=\frac{I_{k} f_{k+1}-I_{k+1} f_{k}}{f_{k+1}-f_{k}} .
$$

Current along $k$-th segment can now be subjected to the analytical inverse Fourier transform:

$$
\tilde{i}_{k}(t)=\int_{-\infty}^{\infty} \tilde{I}_{k}(f) e^{j 2 \pi f t} d f
$$

In order to obtain real time domain counterpart of the current function, the additional term, corresponding to the negative frequencies, is required:

$$
\tilde{i}_{k}(t)=\int_{-f_{k+1}}^{-f_{k}} \tilde{I}_{k}^{*}(-f) e^{j 2 \pi f t} d f+\int_{f_{k}}^{f_{k+1}} \tilde{I}_{k}(f) e^{j 2 \pi f t} d f,
$$

where $\tilde{I}_{k}^{*}(f)$ is complex conjugate of the $\tilde{I}_{k}(f)$.

Analytical solution of integrals (13) is given in a simple closed-form expression, as follows:

$$
\begin{aligned}
\tilde{i}_{k}(t)= & \frac{\left|A_{k}\right|}{2(\pi t)^{2}}\left(\cos \phi_{2}-\cos \phi_{1}\right) \\
& +\frac{\left|A_{k}\right|}{\pi t}\left(f_{2} \sin \phi_{2}-f_{1} \sin \phi_{1}\right), \\
& +\frac{\left|B_{k}\right|}{\pi t}\left(f_{2} \sin \phi_{2}^{\prime}-f_{1} \sin \phi_{1}^{\prime}\right)
\end{aligned}
$$

where

$$
\begin{array}{ll}
\phi_{1}=2 \pi f_{k} t+\arg \left(A_{k}\right) ; & \phi_{2}=2 \pi f_{k+1} t+\arg \left(A_{k}\right) \\
\phi_{1}^{\prime}=2 \pi f_{k} t+\arg \left(B_{k}\right) ; & \phi_{2}^{\prime}=2 \pi f_{k+1} t+\arg \left(B_{k}\right)
\end{array},
$$

Applying relation (14) to all $K_{m}$ segments and summing up the results yields an approximate value of the current at instant $t$ for the $m$-th iteration:

$$
\tilde{i}^{m}(t)=\sum_{k=1}^{K_{m}} \tilde{i}_{k}^{m}(t),
$$

where $\tilde{i}_{k}^{m}(t)$ is the approximate value of current at the instant $t$ along the $k$-th segment for $m$-th iteration.

This simple procedure is particularly convenient for iterative algorithms, where the repeated inversion is required, because the entire sum need not be evaluated. Specifically, in the next (that is, $m+1$ ) iteration, only the segments associated with the newly added frequencies need to be evaluated. Each new frequency determined by the adaptive sampling algorithm as described in previous section, generates two new segments that approximate the actual function better than single segment from the previous (that is, $m$-th) iteration.

For example, if one of the newly added frequencies $f^{N}$ is found between $f_{k}$ and $f_{k+1}$ such that: 


$$
f^{N}=\frac{f_{k}+f_{k+1}}{2}
$$

the $k$-th segment used in previous, $m$-th, iteration will be replaced by the segments $k^{\prime}$ and $k^{\prime \prime}$, as shown in Fig. 3.

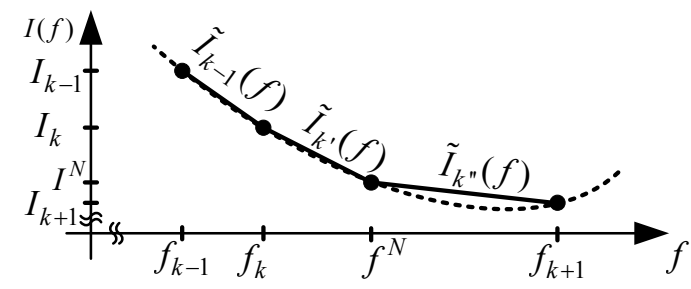

Fig. 3. Adding a new frequency splits the segment from previous

Time domain current for the $m$-th iteration can now be calculated using only contributions from the changed segments. In the example depicted by Fig. 3 , the $k$-th segment from $m-1$ iteration is replaced by segments $k^{\prime}$ and $k^{\prime \prime}$, therefore only the contributions of those segments need to be calculated in order to determine time domain current at $m$-th iteration:

$$
i^{m}(t)=i^{m-1}(t)-i_{k}^{m-1}(t)+i_{k^{\prime}}^{m}(t)+i_{k^{\prime \prime}}^{m}(t) .
$$

Therefore, for each new frequency point added by the adaptive sampling algorithm, only two evaluations of the (14) are required in order to obtain the time domain results. This procedure is repeated for all newly added frequencies in the each iteration.

The principle advantages of this approach, as opposed to using standard inverse FFT, is its simplicity and avoidance of the time discretisation, since the current can be calculated for arbitrary chosen time instants. This can be particularly useful in obtaining the transient response of the structure where the very early times behavior is of interest. In such cases, using standard inverse FFT necessitates the sampling of a very wide frequency spectrum and very high number of samples, which might prove to be impractical, particularly with respect to the algorithm memory requirements. Additionally, unlike inverse FFT, the frequency samples need not be uniformly distributed, which reduces the number of samples during the frequency to time inversion itself. This approach is naturally adapted to the sampling algorithm described in the previous chapter, since the frequency set obtained during adaptive sampling can be directly used in inversion, without further resampling.

The reduction of the number of samples in both frequency and time domain can lead to increased computational efficiency of the frequency to time domain inversion when compared with inverse FFT. If $N$ is a number of frequency and time domain samples, then the inverse FFT will require approximately $N \cdot \log _{2}(N)$ operations. If the analytical inverse Fourier transform is used however, the overall number of basic operations will be approximately $2 N_{F} \cdot N_{T}$, where $N_{F}$ and $N_{T}$ are number of frequency and time domain samples, respectively, and the single basic operation consist of evaluation of relation (14). The number of frequency samples $N_{F}$ is typically significantly smaller then $N$, since $N_{F}$ includes only frequencies selected during adaptive sampling process. The number of time domain samples $N_{T}$ can be chosen arbitrarily. Therefore, if $N_{F}$ and $N_{T}$ are sufficiently smaller then $N$, so that

$$
2 N_{F} \cdot N_{T}<N \cdot \log _{2}(N)
$$

this approach can be more computationally efficient then standard inverse FFT.

Except for the cases of very large number of frequency domain samples $N$, or very small number of time domain samples $N_{T}$, the condition (19) will typically not be satisfied and the FFT approach will be a more efficient one. However, if frequency to time domain inversion is to be performed during each iteration during the adaptive sampling procedure, the complete inverse FFT has to be repeated each time the transfer function is refined, while the proposed approach requires evaluation of only changed segments of the transfer function, resulting in the same overall number of operations $2 N_{F} \cdot N_{T}$, irrespective of the number of iterations during the adaptive sampling algorithm Therefore, using the analytical inverse Fourier transform instead of inverse FFT in this case will be more computationally efficient if following condition is satisfied:

$$
2 N_{F} \cdot N_{T}<K \cdot N \cdot \log _{2}(N),
$$

where $K$ is the number of iterations during the adaptive sampling algorithm.

Finally it should be stressed out that the computational time considerations are of less importance for the efficiency of the overall algorithm. The reason for this is that the computational time cost of the frequency to time domain inversion is typically several orders of magnitude smaller then frequency domain computation. Therefore only a small fraction of the overall computational time is spent on frequency to time domain inversion, irrespective of the inversion technique used.

\section{NUMERICAL RESULTS}

Scattering from two different thin wire geometries, depicted on Fig. 4, is analyzed. In both cases, wires are illuminated by the normally incident EMP plane wave, with the waveform described by the double exponential function:

$$
E_{x}^{i n c}(t)=E_{0}\left(e^{-\alpha t}-e^{-\beta t}\right),
$$

where

$$
E_{0}=1 \mathrm{~V} / \mathrm{m} ; \quad \alpha=4 \cdot 10^{7} \mathrm{~s}^{-1} ; \quad \beta=6 \cdot 10^{8} \mathrm{~s}^{-1} .
$$

Geometry (a), shown on Fig. 4. (a), consist of a single wire of 
length $L=1 \mathrm{~m}$, radius $a=2 \mathrm{~mm}$, placed at height $h=0.5 \mathrm{~m}$ above infinite perfectly conducing plane.

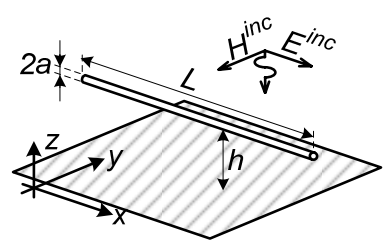

(a)

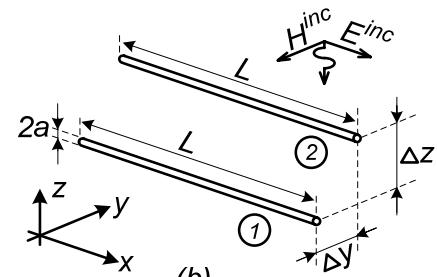

(b)
This can be clearly observed in Figs 8-9, which depict relative error of the results obtained via uniform and adaptive sampling as a function of the total number of samples $N$.

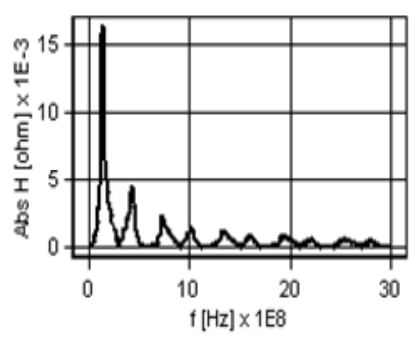

(a) (b)

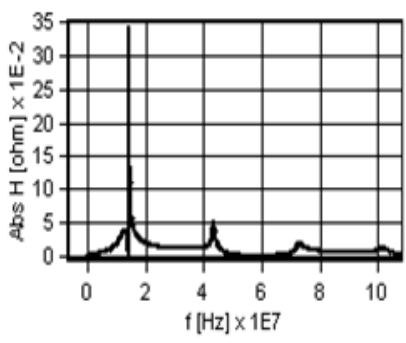

Second analyzed geometry, geometry (b), consist of two wires of the same length $L=10 \mathrm{~m}$ and radius $a=2 \mathrm{~cm}$, placed in free space, as shown on Fig. 4. (b). The wires are displaced relative to each other with the parameters $\Delta y=1 \mathrm{~m}, \Delta z=1 \mathrm{~m}$.

The results are compared with the referent results obtained by Galerkin-Bubnov Boundary Integral Equation Method (GB-BIEM) [17], which is the direct time domain method that has proven to be rather accurate for straight wire geometries [2]. The referent results for the time-domain currents at the center of the wire obtained by GB-BIEM are shown in Figs. 5-6 for geometry (a) and (b), respectively.

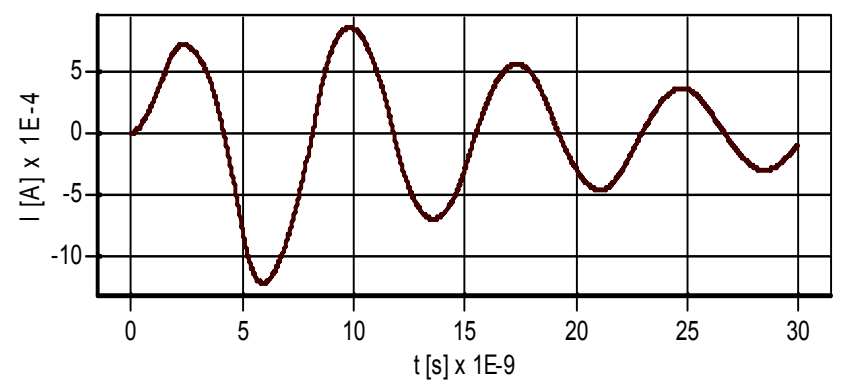

Fig. 5. Current at the center of the wire, geometry (a)

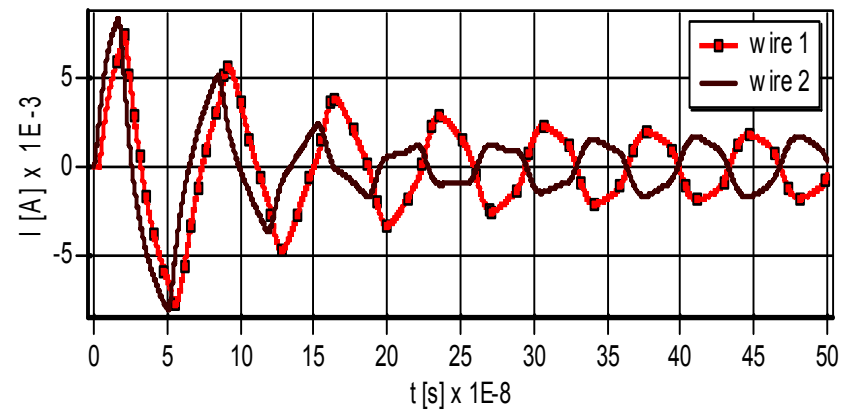

Fig. 6. Current at the center of the wires, geometry (b)

When performing indirect time domain analysis, the transfer functions of the both geometries are obtained, as depicted in Fig. 7. It can be observed that the geometry (b) is highly resonant when compared with geometry (a). The use of the adaptive sampling can therefore be expected to have more impact in this case, then if simple structure, without the highly pronounced peaks in its transfer function, is analyzed.
Fig. 7. Absolute value of the transfer function $H(f)$ for geometry (a) and wire 2 of geometry (b)

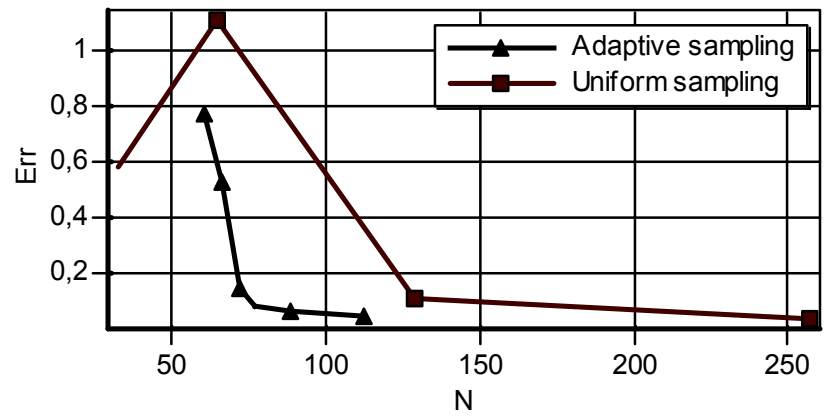

Fig. 8. Relative error to reference results for uniform and adaptive sampling as a function of $N$ for geometry (a)

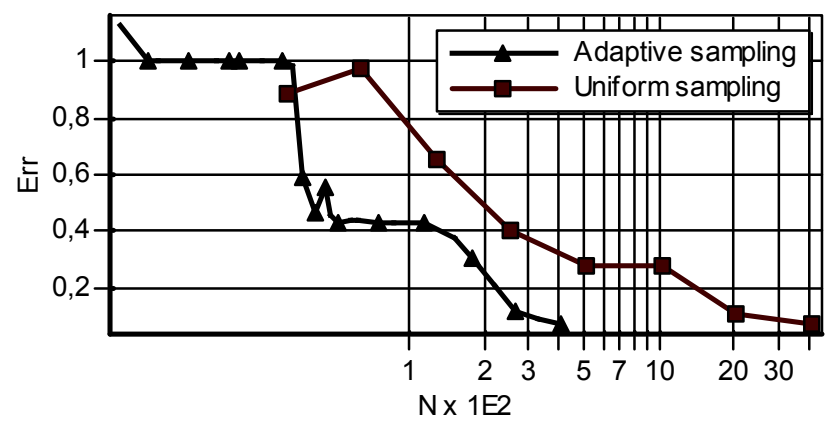

Fig. 9. Relative error to reference results for uniform and adaptive sampling as a function of $N$ for geometry (b), wire 2

Figs. 8-9 are obtained by evaluating the current at the centre of the wire for different number of samples using adaptive and uniform sampling and calculating the relative error to the referent (GB-BIEM) results according to the following expression:

$$
E r r=\frac{\int_{0}^{T}\left|i_{N E C}(t)-i_{B E M}(t)\right| d t}{\int_{0}^{T}\left|i_{B E M}(t)\right| d t} .
$$


where $T$ is the observation period, $i_{B E M}(t)$ is the referent time domain current, and $i_{N E C}(t)$ is the current obtained for the same wire point using NEC in combination with frequency to time domain inversion. The integrals in (23) are numerically evaluated using different number of samples $N$, via uniform, and then adaptive sampling.

When compared to simple uniform sampling, the adaptive sampling algorithm reduces the total number of samples by a factor of two in the case of geometry (a), as depicted by Fig. 8. However, if the geometry (b) (which is highly resonant, as illustrated in Fig. 7 (b)) almost tenfold decrease in the number of samples can be expected if adaptive sampling is used, as illustrated in Fig. 9.

TABLE I

TOTAL NUMBER OF OPERATIONS: INV. ANALYTIC FT VS. INV. FFT

\begin{tabular}{crrrrr}
\hline \hline & \multicolumn{2}{c}{ Geometry (a) } & & \multicolumn{2}{c}{ Geometry (b) } \\
\cline { 2 - 3 } \cline { 5 - 6 } & $\begin{array}{c}\text { Inverse } \\
\text { FFT }\end{array}$ & $\begin{array}{c}\text { Analytic } \\
\text { Inverse } \\
\text { FT }\end{array}$ & & $\begin{array}{c}\text { Inverse } \\
\text { FFT }\end{array}$ & $\begin{array}{c}\text { Analytic } \\
\text { Inverse } \\
\text { FT }\end{array}$ \\
\hline$N_{F}$ & 512 & 113 & & 8.192 & 326 \\
$N_{T}$ & 512 & 180 & & 8.192 & 301 \\
$K$ & 6 & 6 & & 27 & 27 \\
\hline$N_{T O T}$ & 27.648 & 40.680 & & 2.875 .39 & 196.252
\end{tabular}

The comparison of the total estimated number of operations $N_{T O T}$ for both geometries are depicted in Table I. In case the analytic inverse Fourier transform is considered, the number of frequency samples is given by $N_{F}$, while the number of time samples $N_{T}$, as already mentioned, can be arbitrarily chosen. In this case, $N_{T}$ was chosen sufficiently high to generate graphical depiction of the time domain currents illustrated by Figs. 5, 6. The total number of operations is estimated using following expression:

$$
N_{T O T}=2 N_{F} \cdot N_{T} .
$$

In case the inverse FFT is considered, the number of frequency samples is the same as the number of time samples, that is $N=N_{F}=N_{T}$. The number of iterations during the adaptive sampling is given by $K$. The total number of operations $N_{\text {TOT }}$ is multiplied by $K$, since in this case the frequency to time domain inversion is performed after each iteration:

$$
N_{\text {TOT }}=K \cdot N \cdot \log _{2}(N)
$$

Table I displays the relevant data for both inverse analytic Fourier transform and the inverse FFT for both geometries. By comparing the two approaches, it can be observed that inverse FFT takes almost half of the overall number of operations in case geometry (a) is being analyzed. However, in case of geometry (b), the number of operations increases by more than a tenfold in case inverse FFT is used. This is mainly due to the significant reduction of the number of frequency samples $N_{F}$, which directly determines the computational time cost of the analytic inverse Fourier transform. If inverse FFT is used instead, the transfer function has to be resampled densely enough using interpolation, regardless of the efficiency gains resulting from the adaptive sampling. However, it should be noted that the data displayed in Table I pertains to the case when frequency to time domain inversion is performed with each iteration during the adaptive sampling. As previously mentioned, this is convenient, but not critical to the adaptive sampling algorithm. In case the inversion is performed only once instead, the overall number of operations of the inverse FFT will be reduced by a factor of $K$. In this case inverse FFT is more computationally efficient even in case of the geometry (b).

\section{V.CONCLUSION}

In this work previously published adaptive sampling algorithm is further modified to obtain the time domain results from the frequency domain results, using the analytic inverse Fourier transform instead of inverse FFT. Unlike FFT, the derived analytical expression need not be evaluated for the complete frequency domain results, but only for the frequencies changed during each iteration, which makes this approach more suitable for the use with iterative algorithm. The expressions are valid for arbitrary time instants, making the proposed approach particularly useful for geometries where a very early times of the geometry transient response are of interest, and allowing the choice of arbitrary small set of time samples. These advantages can also lead to increased efficiency of the frequency to time domain inversion when compared to inverse FFT, depending on the geometry that is being analyzed. However, the overall performance differences are not significant, since calculation time requirements of inverse FFT during each iteration is typically negligible to the overall iteration calculation time. The principle advantage of using the simple analytical expression instead of the inverse FFT is much easier formulation and implementation within the existing adaptive sampling algorithm.

\section{REFERENCES}

[1] E.K.Miller, J.A.Landt, "Direct time-domain techniques for transient radiation and scattering from wires", IEEE Transactions on Antennas and Propagation, vol. 68, no. 11, pp 1396-1424, Nov. 1980.

[2] D. Poljak, Advanced Modeling in Computational Electromagnetic Compatibility, New York: John Wiley and Sons, 2007.

[3] G.J.Burke, A.J.Poggio, Numerical Electromagnetics Code (NEC) Method of Moments, Part II: Program Description - Code, UCID 18834, Lawrence Livermore Laboratory, January 1981.

[4] S.Tkatchenko, F.Rachidi, and M .Ianoz, " Electromagnetic field coupling to a line of finite length: theory and fast iterative solutions in frequency and time domains", IEEE Transactions on Electromagnetic Compatibility, vol. 37, no. 4, pp. 509-518, Nov 1995.

[5] P. Harms, R. Mittra, and J. Nadolny, "Simulating Measurements for a Cable Radiation Study", IEEE Transactions on Electromagnetic Compatibility, vol. 38, no. 1, pp. 25-30, Feb 1996.

[6] S.Antonijević et al., "Comparison of Time-Domain Thin-Wire Array Current Distribution Calculation Using GB-BIEM and NEC", SoftCOM 2004, Conference proceedings, Softcom library, pp. 194-198 
[7] E.K.Miller, " Using adaptive sampling to minimize the number of samples needed to represent a transfer function", Antennas and Propagation Society International Symposium, pp 588-591, 1996.

[8] C.Y.Tham et al., "A Dynamic Adaptive Sampling Technique in Frequency-Domain Transient Analysis", IEEE Transactions on Electromagnetic Compatibility, vol. 44, no. 4, pp. 522-528, Nov 2002.

[9] S.Wang, X.Guan, D.Wang, X.Ma, Yi S., "Adaptive Selection of Sampling Interval In Frequency Domain for Estimating the Poles of Scatterers", PIERS Online, vol. 3, no. 1, pp. 106-110, 2007.

[10] S.Antonijević, D.Poljak, J.Radić, "Optimized indirect time-domain analysis of the thin-wire structures", SoftCOM 2009, Conference proceedings, Softcom library, pp. 194-198, 2009.

[11] E.O. Brigham, The Fast Fourier Transform and its Applications, Englewood Cliffs, NJ: Prentice-Hall, 1988.

[12] A.G. Tijhuis, Zq. Peng, and A.R. Bretones, "Transient excitation of a straight thin-wire segment: A new look at an old problem," IEEE Transactions on Antennas and Propagation, vol. 40, pp. 1132-1146, Oct. 1992.

[13] H.Bagci, A.E.Yilmaz, V.Lomakin, and E.Michielssen, "Fast solution of mixed-potential time-domain integral equations for half-space environments", IEEE Transactions on Geoscience and Remote Sensing,, vol. 43, no 2., pp. 269-279, Feb. 2005.

[14] E.J.Rothwell, J.Baker, K.M.Chen, and D.P.Nyquist, "Approximate natural response of an arbitrarily shaped thin wire scatterer", IEEE Transactions on Antennas and Propagation, vol. 39, no. 10, pp. 1457 1462, Oct. 1991.

[15] E.K.Miller and J.A.Landt, Direct time-domain techniques for transient radiation and scattering, Lawrence Livermore Laboratory, Interaction Notes, Note 334, July 1976.

[16] F.Tesche, "On the analysis of scattering and antenna problems using the singularity expansion technique", IEEE Transactions on Antennas and Propagation, vol. 21, no. 1, pp. 53-62, Jan. 1973.

[17] D.Poljak, C.A.Brebbia, "Indirect Galerkin-Bubnov boundary element method for solving integral equations in electromagnetics", Engineering Analysis with Boundary Elements, vol.28, no.7, pp.771-777, 2004. 